\title{
Stiripentol protects against calcium oxalate nephrolithiasis and ethylene glycol poisoning
}

\author{
Marine Le Dudal, ${ }^{1,2,3}$ Léa Huguet, ${ }^{1,2}$ Joëlle Perez, ${ }^{1,2}$ Sophie Vandermeersch, ${ }^{1,2}$ Elise Bouderlique, ${ }^{1,2}$ Ellie Tang, ${ }^{1,2}$ Carole Martori, ${ }^{4}$ \\ Nicole Chemaly, ${ }^{5}$ Rima Nabbout, ${ }^{5}$ Jean-Philippe Haymann, ${ }^{1,2,4}$ Vincent Frochot, ${ }^{4}$ Laurent Baud, ${ }^{1,2,4}$ Georges Deschênes, ${ }^{6}$ \\ Michel Daudon, ${ }^{1,2,4}$ and Emmanuel Letavernier ${ }^{1,2,4}$ \\ 'Sorbonne Universités, UPMC Univ Paris 06, UMR S 1155, Paris, France. ${ }^{2}$ INSERM, UMR S 1155, Paris, France. ²Department of Pathology, Ecole Nationale Vétérinaire d'Alfort, Université Paris-Est, \\ Maisons-Alfort, France. ${ }^{4}$ Physiology Unit, AP-HP, Hôpital Tenon, Paris, France. ${ }^{5}$ Pediatric Neurology Unit, Hôpital Necker, AP-HP and Paris Descartes University, Paris, France. \\ ${ }^{6}$ Pediatric Nephrology Unit, Hôpital Robert Debré, AP-HP and Paris Diderot University, Paris, France.
}

Increased urinary oxalate excretion (hyperoxaluria) promotes the formation of calcium oxalate crystals. Monogenic diseases due to hepatic enzyme deficiency result in chronic hyperoxaluria, promoting end-stage renal disease in children and young adults. Ethylene glycol poisoning also results in hyperoxaluria, promoting acute renal failure and frequently death. Stiripentol is an antiepileptic drug used to treat children affected by Dravet syndrome. It has been shown to inhibit neuronal lactate dehydrogenase 5 enzyme. As this isoenzyme is also the last step of hepatic oxalate production, we hypothesized that stiripentol would potentially reduce hepatic oxalate production and urine oxalate excretion. In vitro, stiripentol decreased the synthesis of oxalate by hepatocytes in a dose-dependent manner. In vivo, oral administration of stiripentol significantly reduced urine oxalate excretion in rats. Stiripentol protected the kidneys against calcium oxalate crystal deposits in acute ethylene glycol intoxication and chronic calcium oxalate nephropathy models. In both models, stiripentol significantly improved renal function. Patients affected by Dravet syndrome and treated with stiripentol had a lower urine oxalate excretion than control patients. A young girl affected by severe type I hyperoxaluria received stiripentol for several weeks, and urine oxalate excretion decreased by two-thirds. Stiripentol is a promising potential therapy against genetic hyperoxaluria and ethylene glycol poisoning.

\section{Introduction}

Urine oxalate excretion is low (normal value $<0.5 \mathrm{mmol} / \mathrm{day}$ in humans) but its affinity for calcium ions make it a major promoter of calcium oxalate crystals and kidney stone formation (1). Calcium oxalate precipitation in kidney tubules is particularly fateful when it results from acute intoxication by ethylene glycol or from genetic disorders $(2,3)$. Actually, primary hyperoxaluria is a rare but severe genetic enzymatic defect that increases glyoxylate and oxalate hepatic production. Oxalate precipitates in kidney tubules, leading to end-stage kidney disease and poor prognosis in young patients.

Oxalate is produced in the liver from glyoxylate transformation by lactate dehydrogenase type 5 (LDH5) isoenzyme and excreted in urine, with little biological control (4). To date, there is no drug that reduces oxalate production by the liver. In 2015, Sada et al. demonstrated that stiripentol targets lactate production by LDH5 isoenzymes in neurons in vitro (5). Stiripentol is a safe drug that has been used for years in addition to other antiepileptic

\section{Related Commentary: p. 2201}

Conflict of interest: EL and MD have filed a patent related to the use of stiripentol as a treatment against nephrolithiasis and ethylene glycol poisoning (W02017140658 A1). Copyright: @ 2019, American Society for Clinical Investigation.

Submitted: January 16, 2018; Accepted: April 2, 2019; Published: May 20, 2019.

Reference information: J Clin Invest. 2019;129(6):2571-2577.

https://doi.org/10.1172/JCI99822. drugs to target seizures in Dravet syndrome, a rare form of epilepsy affecting young children $(6,7)$. We hypothesized that stiripentol could also inhibit hepatic LDH5 isoenzymes and oxalate production by the liver, and thereby decrease urinary oxalate excretion.

\section{Results}

Stiripentol decreases $L D H$-mediated oxalate synthesis by hepatocytes in vitro. To investigate whether stiripentol could decrease hepatic oxalate production, hepatocytes (HepG2 cells) were grown in vitro in hydroxyproline-enriched cell culture medium (Figure 1A). The exposure of hepatocytes to stiripentol concentrations at therapeutic levels $(5-100 \mu \mathrm{g} / \mathrm{ml})$ resulted in a dose-dependent and significant decrease in oxalate production $(P=0.03, n=4$ experiments, Figure 1A). To confirm the implication of LDH5 isoenzyme, we performed siRNA experiments targeting LDHA mRNA (LDHA is the subunit of LDH5). siRNA suppressed $60 \%$ to $70 \%$ of $L D H A$ mRNA and significantly decreased oxalate production $(P=0.03$, $n=4$ experiments, Figure $1 \mathrm{~B})$. The addition of $10 \mu \mathrm{g} / \mathrm{ml}$ stiripentol to siRNA mildly decreased oxalate production by HepG 2 cells, suggesting that most of oxalate synthesis was actually due to LDH5 isoenzyme activity (Figure 1B).

Stiripentol decreases urine oxalate excretion in rats. To assess whether stiripentol would also efficiently reduce urine oxalate excretion in vivo, 6 rats received $200 \mathrm{mg} / \mathrm{kg} /$ day stiripentol orally for 48 hours. Urine oxalate/creatinine excretion decreased slightly 

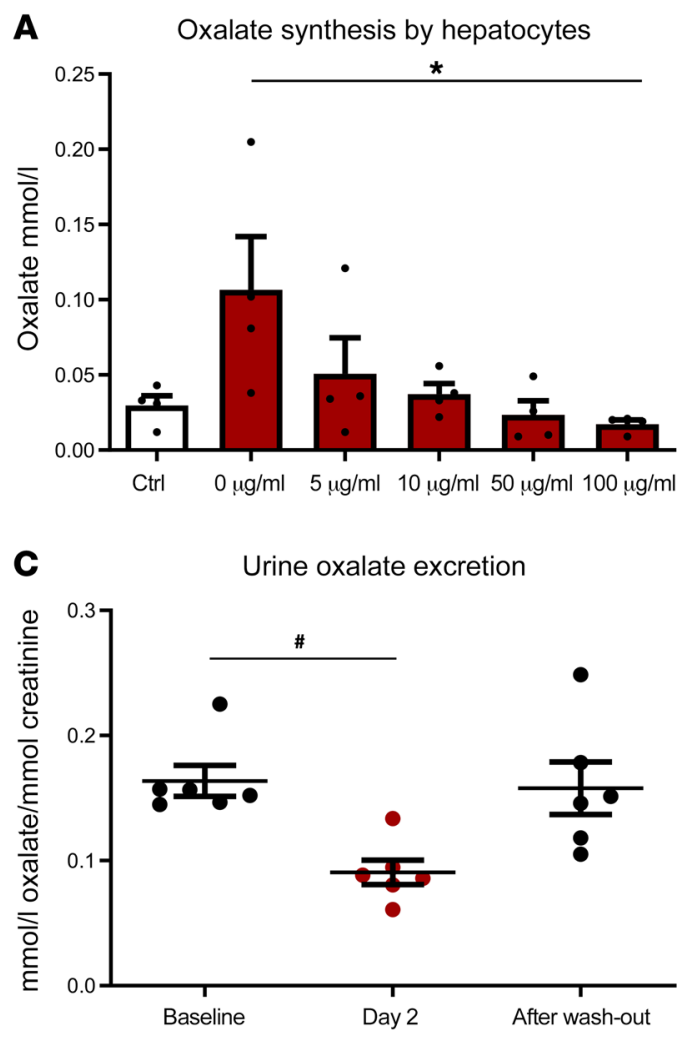

B Oxalate synthesis by hepatocytes

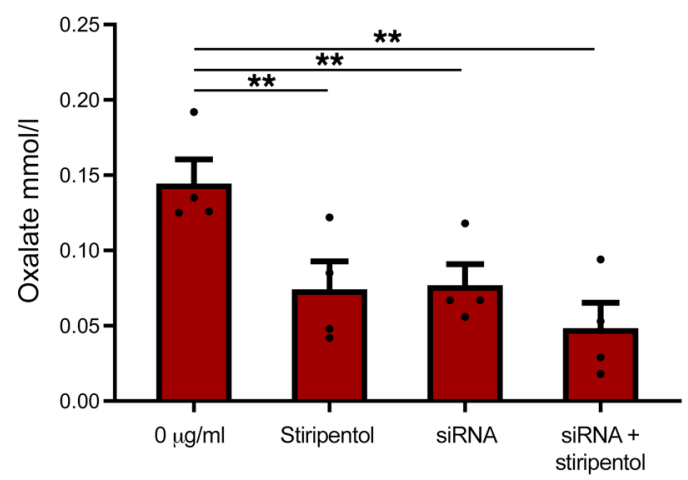

Figure 1. Inhibition of oxalate synthesis by stiripentol in vitro and in vivo. (A) HepG2 cells were grown in a hydroxyproline-enriched medium to produce oxalate (red bars). Oxalate synthesis ( $\mathrm{mM}$ ) was reduced in a dose-dependent manner when stiripentol was added to the medium. ${ }^{*} P=0.03, n=4$ experiments. (B) siRNA targeting $L D H A$ reduced significantly oxalate synthesis and the addition of $10 \mu \mathrm{g} / \mathrm{ml}$ stiripentol to SiRNA reduced mildly oxalate synthesis, suggesting that oxalate synthesis is mostly performed by LDH5. ${ }^{*} P=0.03$ versus control, $n=4$ experiments. (C) Stiripentol given orally for 2 days significantly reduced urine oxalate excretion. ${ }^{*} P=0.002, n=6$ animals. After wash-out, urine oxalate excretion was restored. Data are mean \pm SEM. Mann-Whitney tests (B and C) and Kruskall-Wallis with Dunn's multiple comparison tests (A) were used to compare the different groups. but significantly from $0.16 \pm 0.01 \mathrm{mmol}$ oxalate $/ \mathrm{mmol}$ creatinine before treatment to $0.11 \pm 0.01 \mathrm{mmol}$ oxalate $/ \mathrm{mmol}$ creatinine $(P=0.002)$. After a 4 -day wash-out period, urine oxalate excretion was similar to baseline values (Figure 1C).

Stiripentol protects against ethylene glycol poisoning. Ethylene glycol is metabolized to oxalate and promotes calcium oxalate tubular precipitation. Six rats were exposed to ethylene glycol alone and 6 rats received both ethylene glycol and stiripentol simultaneously at a dose of $300 \mathrm{mg} / \mathrm{kg}$ (and stiripentol in drinking water for the next 2 days). Animals were sacrificed 2 days after ethylene glycol poisoning. Animals exposed to ethylene glycol alone were oliguric, but a small amount of urine was collected at the time of the sacrifice to perform urine crystal analysis. Crystalluria revealed a large number of calcium oxalate monohydrate (COM) crystals in both groups and to a lesser extent calcium oxalate dihydrate crystals (COD), but the mean crystalline volume was 20 times lower in animals receiving stiripentol (9467 $\pm 2259 \mu \mathrm{m}^{3} / \mathrm{mm}^{3}$ versus $201766 \pm 95023 \mu \mathrm{m}^{3} / \mathrm{mm}^{3}, P=0.004$, Figure 2, A and B). The kidneys of rats exposed to ethylene glycol alone were pale and voluminous with a mean weight of 1.31 $\pm 0.11 \mathrm{~g}$, whereas kidneys from animals also receiving stiripentol had a usual red/brown aspect and normal weight at $0.85 \pm 0.02 \mathrm{~g}$ $(P=0.004$, Figure $2 \mathrm{C})$. Stiripentol protected rats against ethylene glycol-induced renal failure (mean serum creatinine 78.2 $\pm 9.6 \mu \mathrm{mol} / 1$ versus $297.1 \pm 74.4 \mu \mathrm{mol} / 1, P=0.002$, Figure $2 \mathrm{G}$ ).

The other biological features are depicted in Table 1 , showing that animals receiving stiripentol had a trend toward lower metabolic acidosis (less decreased bicarbonate serum levels), and a significantly lower serum anion gap, the consequence of lower circulating oxalate levels in this context of ethylene glycol poison- ing. The morphometric analysis of kidney tissues confirmed that stiripentol significantly decreased calcium oxalate crystal deposition in kidneys $(P=0.002$, Figure $2, \mathrm{D}-\mathrm{F})$. These deposits were stained by Yasue coloration (revealing calcium oxalate crystals) and the analysis of kidney tissue by Fourier transform infrared spectroscopy (FTIR) confirmed that crystalline deposits in renal tubules were made exclusively of COM (Figure 2, H and I).

Stiripentol protects against calcium oxalate nephropathy. Sixteen Sprague Dawley rats received hydroxyproline and calcium in drinking water for 16 days to induce calcium oxalate nephropathy. Eight of these rats received stiripentol orally by gavage once a day for 16 days. Urine oxalate excretion increased in both groups after exposure to hydroxyproline but urine oxalate was significantly lower in animals receiving stiripentol in addition to hydroxy-

Table 1. Serum parameters after ethylene glycol intoxication

$\begin{array}{lccc} & \text { EG } & \begin{array}{c}\text { Serum parameters } \\ \text { EG + stiripentol }\end{array} & \boldsymbol{P} \text { value } \\ \text { Creatinine, } \mu \mathrm{mol} / \mathrm{l} & 297.1 \pm 166.3 & 78.1 \pm 21.4 & 0.009 \\ \text { Bicarbonate, } \mathrm{mmol} / \mathrm{l} & 17.7 \pm 4.15 & 20.8 \pm 5.6 & 0.15 \\ \text { Potassium, } \mathrm{mmol} / \mathrm{l} & 6.5 \pm 2.4 & 4.8 \pm 1.3 & 0.07 \\ \text { Sodium, } \mathrm{mmol} / \mathrm{l} & 137.6 \pm 4.7 & 147.4 \pm 3.7 & <0.001 \\ \text { Chloride, } \mathrm{mmol} / \mathrm{l} & 93 \pm 7.9 & 107.6 \pm 9.3 & <0.001 \\ \text { Serum anion Cap, } \mathrm{mmol} / \mathrm{l} & 33.5 \pm 11.1 & 23.7 \pm 12.2 & 0.04\end{array}$

Serum parameters at day 2 after ethylene glycol intoxication ( $n=6$ animals/ group). Data are mean \pm SD. Student bilateral $t$ tests (assuming Gaussian distribution of biological values) were performed. EG, ethylene glycol. 

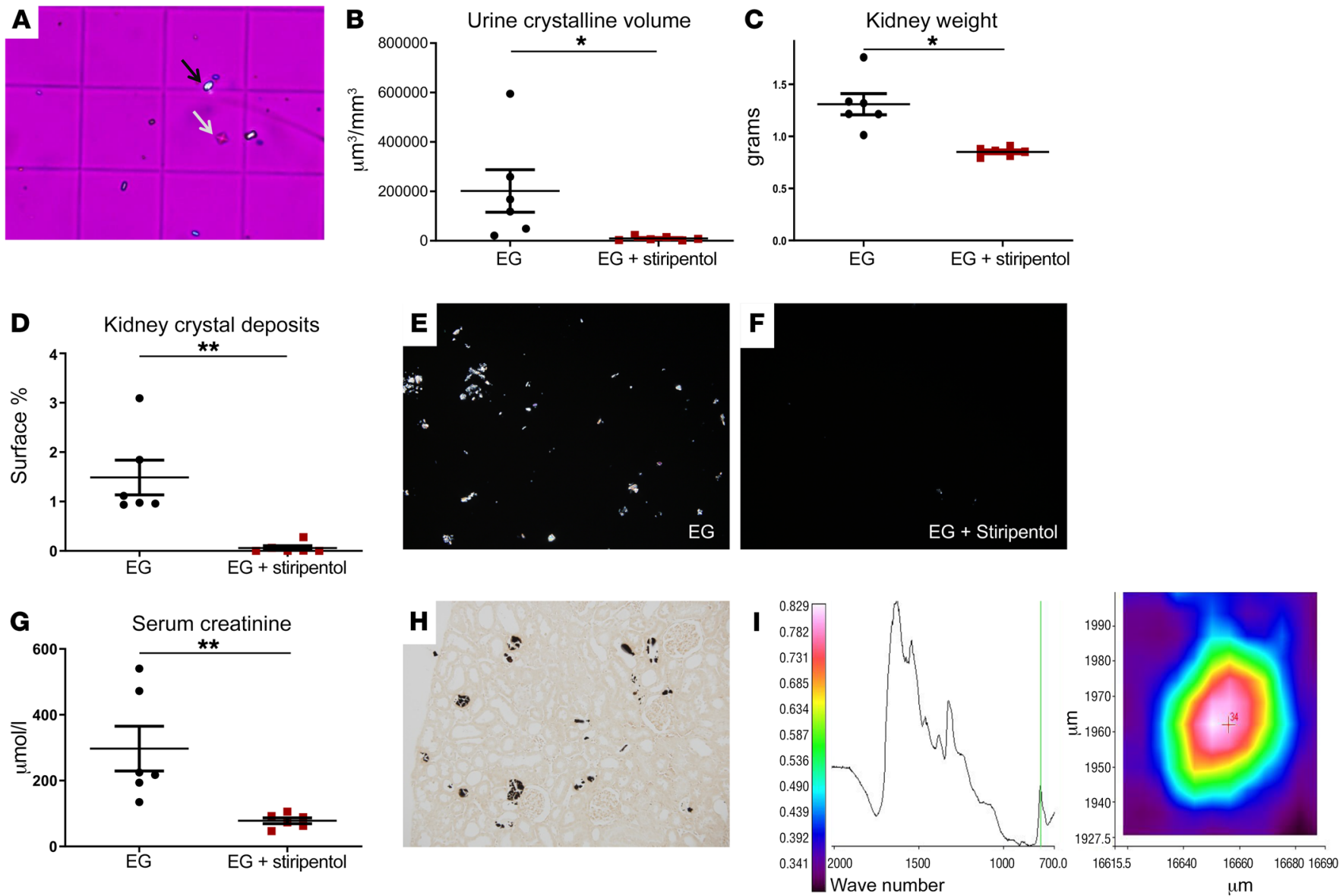

Figure 2. Stiripentol protects against ethylene glycol intoxication. (A) Representative crystalluria showing the presence of calcium oxalate monohydrate crystals (black arrow) and to a lesser extent calcium oxalate dihydrate crystals (white arrow) in urine. Original magnification, $\times 400$. (B) Mean crystalline volume in urine was significantly lower in animals receiving stiripentol in addition to ethylene glycol (EG + stiripentol, red squares) than in animals receiving ethylene glycol alone (EG, black circles). ${ }^{*} P=0.004, n=6$ animals/group). (C) The weight of kidneys from rats exposed to ethylene glycol only was increased when compared with kidneys from rats treated by stiripentol. ${ }^{*} P=0.004, n=6$ ). (D-F) Kidney crystalline accumulation was prevented by stiripentol. ${ }^{* *} P=0.002, n=6$. (G) Stiripentol protected rats against ethylene glycol-induced renal failure. ${ }^{* *} P=0.002, n=6$. $(\mathbf{H})$ Crystalline deposits were stained by Yasue coloration, evidencing their calcic nature. (I) Fourier transform infrared spectroscopy revealed the exclusive presence of COM among tubular deposits. Data are mean \pm SEM. Mann-Whitney tests were used to compare the different groups. Original magnification E, F, H, $\times 200$.

proline and calcium $(P=0.0002$ at day $8, P=0.0019$ at day 11 , $P=0.0002$ at day 15 , Figure 3A). Crystalluria revealed the presence of calcium oxalate crystals in both groups, but the mean crystalline volume was significantly lower in animals treated by stiripentol ( $P=0.015$ at day $8, P=0.007$ at day 15 , Figure 3B). FTIR analysis revealed the presence of sparse deposits of COM crystals in renal tubules. Kidney morphometric analysis demonstrated that stiripentol significantly decreased calcium oxalate deposits in kidney tissues $(P=0.004$, Figure $3, \mathrm{C}-\mathrm{E})$. Renal function was less altered in animals receiving stiripentol $(P=0.028$, Figure $3 \mathrm{~F})$. In parallel, urine glycolate excretion increased in both groups, but it increased significantly more in rats exposed to stiripentol (at days 2 and 8), suggesting that stiripentol actually inhibits the transformation of glyoxylate into oxalate (Figure 4).

Stiripentol decreases urine oxalate excretion in humans. To assess whether stiripentol would efficiently reduce oxalate synthesis in humans, urine oxalate excretion was assessed in children affected by Dravet syndrome who required stiripentol treatment $(n=$ 8 , median age 6 years) and in children of similar age affected by cystinuria who are kidney-stone formers whose stones are not due to oxalate (control group, $n=40$, median age 7.5 years). Urine oxalate excretion was significantly decreased in patients affected by Dravet syndrome, suggesting that stiripentol lowers urine oxalate excretion, even when urine oxalate concentrations stand within normal range $(P=0.002$, Figure $5 \mathrm{~A})$.

A 17-year-old girl affected by severe type I hyperoxaluria (homozygous AGXT mutation c.349-350 insG), and followed by the pediatric nephrology department of Robert Debré Hospital (Paris, France), received stiripentol to reduce urine oxalate excretion. Her renal function was still normal in the absence of an acute episode (serum creatinine $65 \mu \mathrm{mol} / \mathrm{l}$, estimated glomerular filtration rate $88 \mathrm{ml} / \mathrm{min} / 1.73 \mathrm{~m}^{2}$ ), but she was exhausted by recurrent colic nephritis (about once a month), repeated urological procedures, and pyelonephritis. Urine oxalate/creatinine ratio was about $0.18-0.20 \mathrm{mmol}$ oxalate $/ \mathrm{mmol}$ creatinine. Her brother, affected by the same disease, had a rapid decrease in renal function and was treated with combined liver-kidney transplantation. Considering the evolution of the disease, in accordance with the 
A

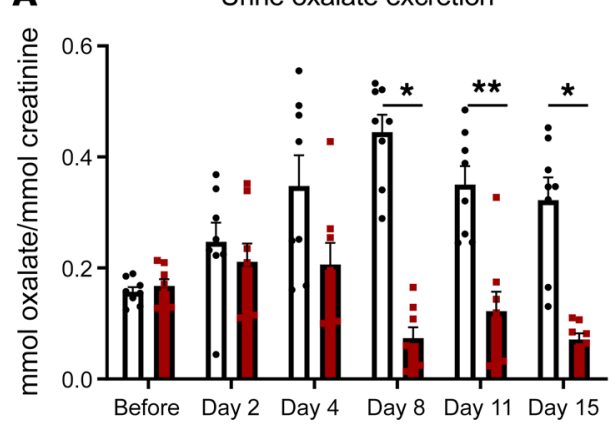

C

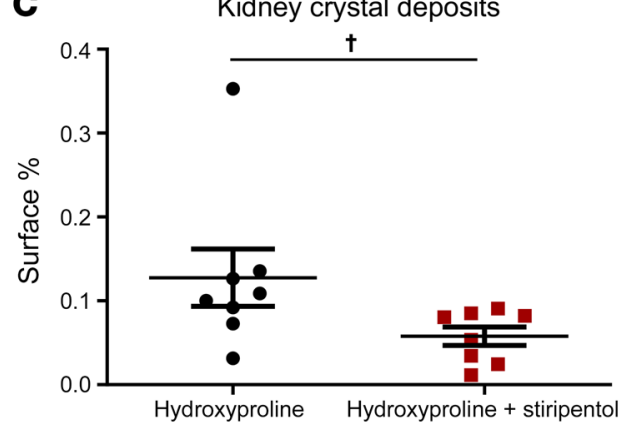

B
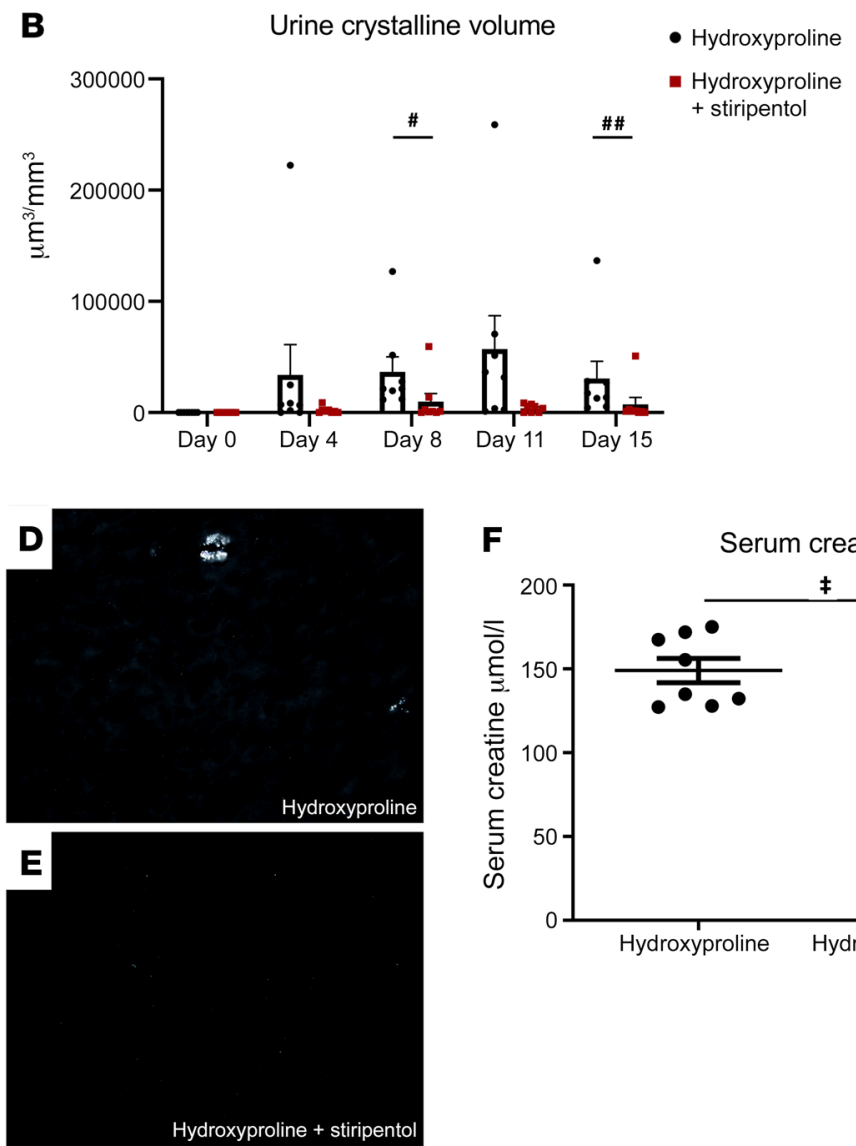

$\mathbf{F}$

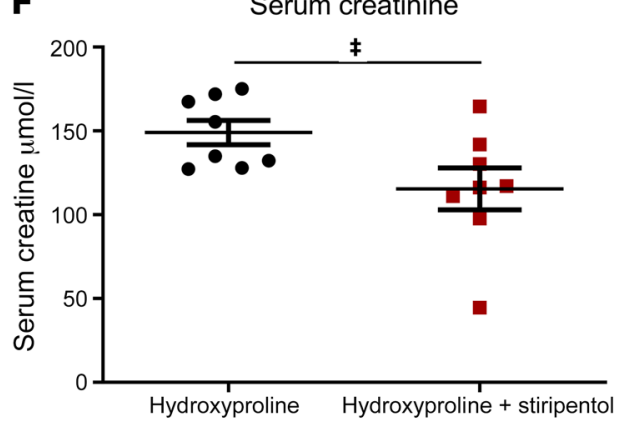

Figure 3. Stiripentol protects against calcium oxalate nephropathy. (A) Urine oxalate excretion was increased by hydroxyproline-enriched diet, and the daily administration of stiripentol (red bars) protected partly against hyperoxaluria. ${ }^{*} P=0.0002$ at days 8 and $15,{ }^{* *} P=0.0019$ at day $11 ; n=8$ animals/

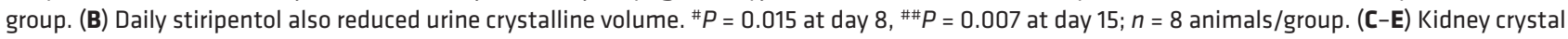
deposits were reduced by stiripentol. ${ }^{\dagger} P=0.004, n=8$ animals/group. (F) Stiripentol protected rats against hydroxyproline-induced renal failure. ${ }^{\ddagger} P=0.028, n=8$ animals/group. Hydroxyproline + stiripentol, red squares; hydroxyproline, black circles. Data are mean \pm SEM. Mann-Whitney and Kruskall-Wallis with Dunn's multiple comparison tests were used to compare the different groups.

medical team and after parental agreement, stiripentol was introduced at a half-dose $(25 \mathrm{mg} / \mathrm{kg} /$ day $)$ and urine oxalate excretion decreased rapidly (Figure 5B). A further decrease in urine oxalate excretion was observed when stiripentol therapy was increased to $50 \mathrm{mg} / \mathrm{kg} /$ day (urine oxalate/creatinine ratio: $0.068 \mathrm{mmol}$ oxalate $/ \mathrm{mmol}$ creatinine), without identified side effects (Figure $5 \mathrm{~B}$ ).

\section{Discussion}

This study shows that stiripentol, an antiepileptic drug, decreases oxalate synthesis by hepatocytes through LDH5 isoenzyme inhibition, and blunts urine oxalate excretion in murine models, protecting against ethylene glycol poisoning and calcium oxalate nephropathy.

The exposure of hepatocytes to stiripentol in vitro at a therapeutic concentration (5 to $100 \mu \mathrm{g} / \mathrm{ml}$ ) resulted in a dose-dependent and significant decrease in oxalate production $(P=0.03)$. As a matter of comparison, the recommended dose of stiripentol in Dravet syndrome is usually $1000-3000 \mathrm{mg} /$ day in children, giving serum levels of $4-22 \mu \mathrm{g} / \mathrm{ml}(6,7)$. As the drug has high first pass metabolism in the liver, it may even be hypothesized that relatively low doses of stiripentol could inhibit hepatic LDH activity efficiently without significant inhibition of systemic LDH5 isoen- zymes. We provide evidence that stiripentol is actually efficient to reduce urine oxalate excretion in epileptic patients affected by Dravet syndrome but also in children affected by primary hyperoxaluria. These results support the hypothesis that stiripentol at the usual dose could be an effective treatment against ethylene glycol intoxication and primary hyperoxaluria.

Ethylene glycol is present in antifreeze and is a frequent cause of poisoning, causing more than 5000 intoxications in the United States each year (2). Its toxicity depends mainly on its transformation into oxalic acid by the liver, which precipitates as calcium oxalate in the kidneys, promoting acute renal failure and acidosis. Ethylene glycol is first metabolized to glycolaldehyde by the enzyme alcohol dehydrogenase, and then undergoes oxidation to glycolate, glyoxylate, and at last oxalate. To date, the specific treatment of ethylene glycol poisoning is fomepizole or 4-methylpyrazole, a competitive inhibitor of alcohol dehydrogenase in the liver (8). The use of stiripentol as a therapy against ethylene glycol intoxication would be of interest since it acts downstream of the metabolic pathway and could therefore still be efficient after a relatively long period following intoxication, even when high amounts of glycolaldehyde have already been produced. 


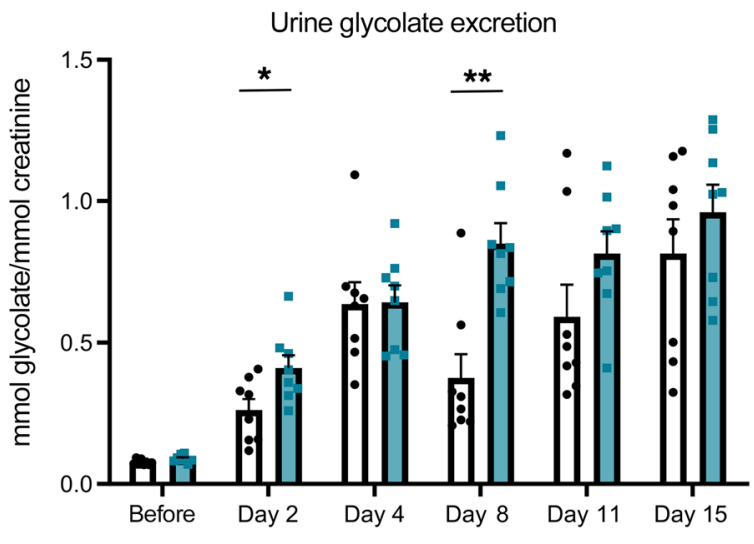

Figure 4. Urine glycolate excretion was increased by a hydroxyprolineenriched diet in rats. At day 2 and day 8 glycolate excretion was significantly more increased in animals exposed to stiripentol than in controls. ${ }^{*} P=0.049$ and ${ }^{* *} P=0.004$, respectively; $n=8$ animals/group. Hydroxyproline, black circles and empty bars; hydroxyproline + stiripentol, blue squares and bars. Data are mean \pm SEM. Kruskall-Wallis with Dunn's multiple comparison tests was used to compare the different groups.

Blocking oxalate synthesis could be of particular interest in primary hyperoxaluria, a genetic enzymatic defect resulting from a mutation of AGXT, GRHPR, or HOGA1 genes, increasing glyoxylate hepatic production which is finally transformed into oxalate by LDH5 (3). Oxalate precipitates in renal tubules, leading to end-stage kidney disease in children and young adults with high morbidity and mortality. The genetic defect may be cured by a liver transplant, but this disabling therapy is usually performed as a combined liverkidney allograft when end-stage renal disease occurs (9). There is currently no drug decreasing oxalate production by the liver with the exception of pyridoxine, which may lower urine oxalate excretion in some cases of type I hyperoxaluria. Administration of hydroxyproline to rats is a classic animal model of hyperoxaluria inducing calcium oxalate nephropathy by increasing hepatic oxalate production as the result of mitochondrial hydroxyproline metabolism (10). This nephropathy is characterized by calcium oxalate intratubular crystal deposits similar to those observed in primary hyperoxaluria (10). The protection against calcium oxalate nephropathy in this model and the dramatic decrease in urine oxalate excretion in a young girl affected by primary hyperoxaluria bring new hope for treating children affected by primary hyperoxaluria.

The main adverse events related to stiripentol in patients with Dravet syndrome are nutrition disorders (loss of appetite, weight loss), neurological disorders (drowsiness, hyperexcitability), and in some rare cases neutropenia. Most side effects disappear when the dose of comedication is decreased (val- proate and clobazepam). Actually, stiripentol interacts with several P450 cytochromes (6). Fortunately, children affected by primary hyperoxaluria are not supposed to receive drugs other than pyridoxine and potassium citrate.

In summary, stiripentol, a potent LDH5 enzyme inhibitor, limits oxalate production by human hepatocytes in vitro and in rats in vivo when used at therapeutic levels. Oral administration of stiripentol protects rats against ethylene glycol poisoning and oxalate nephropathy. The drug brings new hope to prevention of the renal and systemic consequences of primary hyperoxaluria, a disease associated with a dramatic morbidity and mortality, and to the treatment of ethylene glycol poisoning. The safety profile of stiripentol and its preliminary results in children make it deserving of clinical studies.

\section{Methods}

\section{Cell culture}

Cells from HepG2, a hepatoma human cell line (Sigma-Aldrich), were grown in DMEM (Gibco, Invitrogen) supplemented with 10\% FBS (Gibco), 1\% glutamine, $4.5 \mathrm{~g} / \mathrm{l}$ glucose, and $50 \mathrm{U} / \mathrm{ml}$ penicillin/streptomycin (Gibco). HepG2 cell medium was supplemented with $10 \mathrm{mM}$ hydroxyproline to induce oxalate production as previously described (11) and with various concentrations of stiripentol $(5-100 \mu \mathrm{g} / \mathrm{ml}$, Sigma-Aldrich) for 24 hours. To modulate LDH-A expression, HEpG2 cells were transfected with siRNA targeting the LDHA human gene, 4 siRNAs for Entrez gene 3939: FlexiTube siRNA functionally verified SI02663535 and SIO0300622; FlexiTube siRNA SI04949616 and SI04949609; or positive (silencing) control siRNA Mm/Hs MAPK1 or control siRNA SI03650318 (both from Qiagen). Briefly, HepG2 cells were seeded in 100-mm Petri dishes at a density of $4 \times 10^{6}$ cells/Petri dish in $10 \mathrm{ml}$ DMEM and 10\% FBS and directly transfected with $5 \mathrm{nM}$ siRNA through the use of $40 \mu \mathrm{l}$ HiPerFect transfection reagent (Qiagen). Twenty-four hours after transfection, cells were stimulated for 24 hours with hydroxyproline $(10 \mathrm{mM})$ with or without stiripentol $(10 \mu \mathrm{g} / \mathrm{ml})$. Cells were then lysed in RLT, and RNA was isolated with an EZ-10 Spin Column Kit (Proteogenix) and reverse transcribed with a First-Strand cDNA Synthesis Kit (Thermo Fisher Scientific). cDNA was amplified on a Light Cycler 480 system (Roche) using SYBR Green (Roche) and specific primers for LDHA (5'- TCTCTGTAGCAGATTTGGCAGA-3'
A

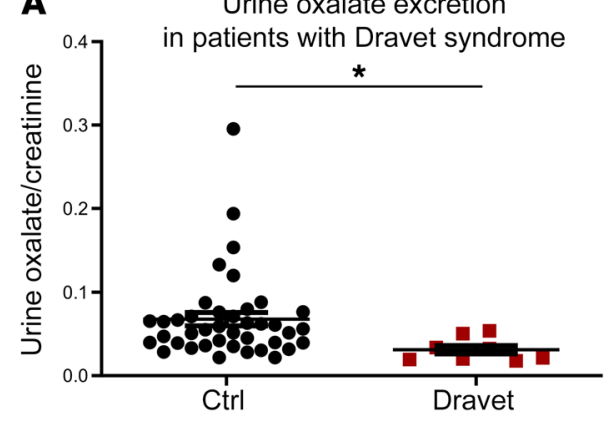

B

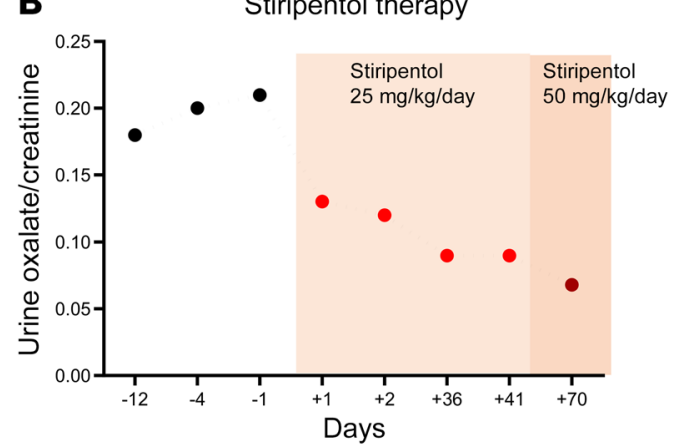

Figure 5. Stiripentol decreases urine oxalate excretion in humans. (A) Urine oxalate excretion (indexed to creatinine, $\mathrm{mmol}$ oxalate/mmol creatinine) was lower in children affected by Dravet syndrome and treated with stiripentol than in controls (i.e., children affected by cystinuria). ${ }^{*} P=0.002$; Dravet + stiripentol, red squares, $n=8$; controls, black circles, $n=40$ ). Mann-Whitney test was used to compare the 2 groups. (B) Urine oxalate excretion (indexed to creatinine, $\mathrm{mmol}$ oxalate/mmol creatinine) was measured before and after stiripentol therapy (25 $\mathrm{mg} /$ $\mathrm{kg} /$ day and $50 \mathrm{mg} / \mathrm{kg} /$ day) in a young girl affected by type I primary hyperoxaluria. 
and 5'-AAGACATCATCCTTTATTCCGTAAA-3'), MAPK1 (5'-TCTGCACCGTGACCTCAA-3' and 5'-GCCAGGCCAAAGTCACAG-3'), and GAPDH (5'-TCCACTGGCGTCTTCACC-3' and 5'-GGCAGAGATGATGACCCTTTT-3') as housekeeping gene.

\section{Animals}

Effect of stiripentol on urine oxalate excretion. Six-week-old SpragueDawley male rats were purchased from Harlan Laboratories. All efforts were made to reduce animal suffering. Mice were housed in similar conditions ( 3 rats/cage) with a 12-hour dark/light cycle and fed ad libitum on standard rat chow.

Rats received 200 mg/kg stiripentol (Diacomit, Biocodex) twice orally by gavage, and urine samples were collected before treatment, 48 hours after treatment initiation and after a wash-out period.

Ethylene glycol intoxication protocol. Six 8-week-old Sprague-Dawley male rats (control group) received one $6 \mathrm{~g} / \mathrm{kg}$ dose of ethylene glycol by gavage (batch no. SHBG0526V, Sigma-Aldrich). Six rats (stiripentol group) received $6 \mathrm{~g} / \mathrm{kg}$ ethylene glycol orally and $300 \mathrm{mg} /$ $\mathrm{kg}$ stiripentol by gavage at the same time. Rats in the stiripentol group had free access to water containing $4 \mathrm{~g} / \mathrm{l}$ stiripentol, whereas rats from the control group had free access to water without stiripentol. All animals were sacrificed 48 hours later.

Oxalate nephropathy protocol. Sixteen 8-week-old Sprague-Dawley male rats had free access to water containing $2 \mathrm{~g} / \mathrm{l}$ calcium (calcium chloride) and $20 \mathrm{~g} / \mathrm{l}$ hydroxyproline (batch no. 090289, Interchim SA) for 16 days. Eight of the mice received $300 \mathrm{mg} / \mathrm{kg}$ stiripentol daily by gavage for 16 days. Environmental enrichment was routinely performed. All animal procedures were performed in accordance with the European Union Guidelines for the Care and Use of Laboratory Animals and in accordance with local Institutional Animal Care and Use Committee ("comité d'éthique en experimentation Charles Darwin C2EA-05") guidelines.

\section{Biological samples and biochemistry}

Ethylene glycol intoxication protocol. A sample of urine was collected before protocol and at the time of sacrifice in the acute model of ethylene glycol poisoning, to assess crystalluria. A blood serum sample was collected before protocol and at the time of sacrifice. The blood samples were analyzed for creatinine by enzymatic assay, bicarbonate, sodium, chloride, and potassium to assess renal function and acidosis, using an iSYS analyzer (Immunodiagnostic Systems) and an ABL815 (Radiometer).

Oxalate nephropathy protocol. Urine was collected before the protocol and at days 2, 4, 8, 11, and 15 for 24 hours from mice in metabolic cages with free access to water (enriched in calcium and hydroxyproline), to assess urine oxalate excretion. Fresh urine was collected after spontaneous voiding to perform crystalluria before the protocol and at days $4,8,11$, and 15 , immediately before the 24 -hour urine collection. The number, size, and type of crystals were analyzed by trained technicians to measure the mean crystalline volume (12). Blood $(1 \mathrm{ml})$ was collected at the time of sacrifice to measure renal function.

Oxalate urinary excretion in humans. A single urine sample was collected from patients in Necker Hospital (Paris, France) who were affected by Dravet syndrome, after parental informed and written consent and ethical committee authorization (no. ID-RCB 2016A01032-49). Urine oxalate excretion in patients affected by cystinuria was obtained from the Tenon hospital (Paris, France) database (CNIL declaration number $1709404 \mathrm{vO}$ ). Urine oxalate was routinely assessed in all kidney stone formers. Urine oxalate excretion in the patient affected by primary hyperoxaluria was collected prospectively before and after stiripentol therapy.

Creatinine was analyzed by enzymatic methods (blood) and Jaffe method (urine) on an iSYS analyzer (Immunodiagnostic Systems). Oxalate and glycolate urine levels were measured by ion chromatography (Dionex ICS-3000).

\section{Histology, Yasue staining, and morphometry}

Kidney tissues were fixed in AFA and formalin and embedded in paraffin. Tissue sections $(4 \mu \mathrm{m})$ were stained by Yasue procedure to reveal tissue calcifications. A morphometric analysis of calcified tissue surface was performed with the Image $\mathrm{J}$ software $(\mathrm{NIH})$ on 5 photographs at $\times 200$ magnification by using polarized light to reveal crystalline deposits.

\section{$\mu \mathrm{FTIR}$ spectroscopy}

Microcalcification phases were characterized using $\mu$ Fourier Transform InfraRed spectrometry. Tissue sections $(4 \mu \mathrm{m})$ were deposited on low emission microscope slides (MirrIR, Keveley Technologies, Tienta Sciences). FT-IR hyperspectral images were recorded with a Spectrum spotlight 400 FT-IR imaging system (Perkin Elmer Life Sciences) with a spatial resolution of $6.25 \mu \mathrm{m}$ and a spectral resolution of $8 \mathrm{~cm}^{-1}$. The spectra were recorded in the $4000-700 \mathrm{~cm}^{-1}$ mid-InfraRed range. Each spectral image, covering a substantial part of the tissue, consisted of about 30,000 spectra.

\section{Statistics}

Data are shown as mean \pm SEM (figures) and mean \pm SD (tables). Mann-Whitney and Kruskall-Wallis with Dunn's multiple comparison tests were used to compare the different groups, with the exception of serum parameters compared with bilateral $t$ tests, using Prism and Statview softwares. The level of significance was set to less than 0.05.

\section{Study approval}

For animal studies, a specific authorization was obtained from the French Research Ministry and from the university ethical committee (no. 51102016042012069009 v2). For human studies, a specific authorization was obtained from the Comité de Protection des Personnes (CPP) d'Ile de France (no. ID-RCB 2016-A01032-49).

\section{Author contributions}

EL, MLD, and MD designed the study. EL, MLD, SV, ET, EB, RN, NC, GD, LH, JP, CM, JPH, VF, LB, and MD conducted experiments. EL, MLD, LH, and MD analyzed data. EL, MD, MLD, and LB wrote the manuscript.

\section{Acknowledgments}

The authors thank Albert Bensman for his help in preparing the final version of the manuscript. This work was supported by the Agence Nationale de la Recherche (ANR-13-JSV1-0010-01), the Académie Nationale de Médecine (Nestlé-Waters Award), Convergence-UPMC CVG1205, and CORDDIM-2013-COD130042.

Address correspondence to: Emmanuel Letavernier, Service des Explorations Fonctionnelles Multidisciplinaires, Hôpital Tenon, 4 rue de la Chine, 75020 Paris, France. Phone: 33.1.56.01.67.73; Email: emmanuel.letavernier@aphp.fr. 
1. Khan SR, et al. Kidney stones. Nat Rev Dis Primers. 2016;2:16008.

2. Kruse JA. Methanol and ethylene glycol intoxication. Crit Care Clin. 2012;28(4):661-711.

3. Cochat P, Rumsby G. Primary hyperoxaluria. NEngl J Med. 2013;369(7):649-658.

4. Holmes RP, Assimos DG. Glyoxylate synthesis, and its modulation and influence on oxalate synthesis. JUrol. 1998;160(5):1617-1624.

5. Sada N, Lee S, Katsu T, Otsuki T, Inoue T. Epilepsy treatment. Targeting LDH enzymes with a stiripentol analog to treat epilepsy. Science. 2015;347(6228):1362-1367.
6. Verrotti A, Prezioso G, Stagi S, Paolino MC, Parisi P. Pharmacological considerations in the use of stiripentol for the treatment of epilepsy. Expert Opin Drug Metab Toxicol. 2016;12(3):345-352.

7. Farwell JR, Anderson GD, Kerr BM, Tor JA, Levy RH. stiripentol in atypical absence seizures in children: an open trial. Epilepsia. 1993;34(2):305-311.

8. Hall TL. Fomepizole in the treatment of ethylene glycol poisoning. CJEM. 2002;4(3):199-204.

9. Bergstralh EJ, et al. Transplantation outcomes in primary hyperoxaluria. Am J Transplant.
2010;10(11):2493-2501.

10. Khan SR, Glenton PA, Byer KJ. Modeling of hyperoxaluric calcium oxalate nephrolithiasis: experimental induction of hyperoxaluria by hydroxy-Lproline. Kidney Int. 2006;70(5):914-923.

11. Baker PR, Cramer SD, Kennedy M, Assimos DG, Holmes RP. Glycolate and glyoxylate metabolism in HepG2 cells. Am J Physiol Cell Physiol. 2004;287(5):C1359-C1365.

12. Daudon M, Hennequin C, Boujelben G, Lacour B, Jungers P. Serial crystalluria determination and the risk of recurrence in calcium stone formers. Kidney Int. 2005;67(5):1934-1943. 